This document is confidential and is proprietary to the American Chemical Society and its authors. Do not copy or disclose without written permission. If you have received this item in error, notify the sender and delete all copies.

\title{
Identification of Luminescence Centers in Molecular-Sized Silicon Carbide Nanocrystals
}

\begin{tabular}{|c|c|}
\hline Journal: & The Journal of Physical Chemistry \\
\hline Manuscript ID & jp-2015-095033 \\
\hline Manuscript Type: & Article \\
\hline Date Submitted by the Author: & 29-Sep-2015 \\
\hline Complete List of Authors: & $\begin{array}{l}\text { Beke, Dávid; Wigner Research Centre for Physics, Institute for Solid State } \\
\text { Physics and Optics, Hungarian Academy of Sciences; Budapest University } \\
\text { of Technology and Economics, Faculty of Chemical Technology and } \\
\text { Biotechnology } \\
\text { Jánosi, Tibor; University of Pécs, Institute of Physics; University of Pécs, } \\
\text { Szentágothai Research Center, Spectroscopy Research Group } \\
\text { Somogyi, Bálint; Wigner Research Centre for Physics, Institute for Solid } \\
\text { State Physics and Optics, Hungarian Academy of Sciences, } \\
\text { Major, Dániel; Budapest University of Technology and Economics, Faculty } \\
\text { of Chemical Technology and Biotechnology } \\
\text { Szekrényes, Zsolt; Research Institute for Solid State Physics and Optics, } \\
\text { Erostyák, János; University of Pécs, Institute of Physics; University of Pécs, } \\
\text { Szentágothai Research Centre } \\
\text { Kamarás, Katalin; Wigner Research Centre for Physics, Institute for Solid } \\
\text { State Physics and Optics } \\
\text { Gali, Adam; Wigner Research Centre for Physics , Institute of Solid State } \\
\text { Physics and Optics }\end{array}$ \\
\hline
\end{tabular}




\title{
Identification of Luminescence Centers in Molecular-
}

\section{Sized Silicon Carbide Nanocrystals}

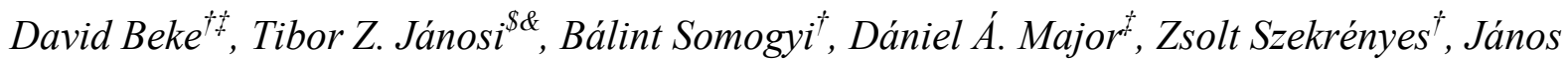 \\ Erostyák $^{\$ \&}$, Katalin Kamarás ${ }^{\dagger}$, Adam Gali ${ }^{\dagger *} *$ \\ $\uparrow$ Institute for Solid State Physics and Optics, Wigner Research Centre for Physics, Hungarian Academy \\ of Sciences, PO. Box 49, H-1525 Budapest, Hungary \\ \$ Faculty of Chemical Technology and Biotechnology, Budapest University of Technology and Eco- \\ nomics, H-1111 Müegyetem rkp. 3., H-1111 Budapest, Hungary \\ \$ University of Pécs, Institute of Physics, Ifjúság útja 6., H-7624, Pécs, Hungary
}

\& University of Pécs, Szentágothai Research Center, Spectroscopy Research Group, Ifjúság útja 20., H7624, Pécs, Hungary

\# Department of Atomic Physics, Budapest University of Technology and Economics, Budafoki út 8, H1111 Budapest, Hungary

KEYWORDS: Silicon Carbide, Quantum Dots, Luminescence, TRES, DAS, Surface Chemistry, Nanocrystal, Colloid

\begin{abstract}
Understanding the fluorescence of complex systems such as small nanocrystals with various surface terminations in solution is still a scientific challenge. Here we show that the combination of advanced time-resolved spectroscopy and ab initio simulations, aided by surface engineering, is able to


identify the luminescence centers of such complex systems. Fluorescent water soluble silicon carbide (SiC) nanocrystals have been previously identified as complex molecular systems of silicon, carbon, oxygen and hydrogen held together by covalent bonds that made the identification of their luminescence centers unambiguous. The aqueous solutions of molecular-sized SiC nanocrystals are exceedingly promising candidates to realize bioinert non-perturbative fluorescent nanoparticles for in vivo bioimaging, thus the identification of their luminescent centers is of immediate interest. Here we present identification of two emission centers of this complex system: surface groups involving carbon - oxygen bonds and a defect consisting of silicon - oxygen bonds which becomes the dominant pathway for radiative decay after total reduction of the surface. The identification of these luminescent centers reconciles previous experimental results on the surface and $\mathrm{pH}$ dependent emission of $\mathrm{SiC}$ nanocrystals and helps design optimized fluorophores and nanosensors for in vivo bioimaging.

\section{Introduction}

Silicon carbide $(\mathrm{SiC})$ is a wide band gap indirect semiconductor ${ }^{1}$ with a variety of applications such as high power electronics, spintronics ${ }^{2}$ and quantum information processing ${ }^{3-7}$. SiC nanocrystals (NCs) are proven to be favorable biological labels due to their good biocompatibility ${ }^{8,9}$, hemocompatibility ${ }^{10}$ and excellent solubility in polar solvents ${ }^{11}$. Moreover, they contain many surface groups that are suitable for further chemical modifications for targeted biomolecules ${ }^{12}$. Even though the applicability of SiC NCs in biological environment ${ }^{9,13}$ and therapy ${ }^{14}$ was demonstrated, understanding the connection between the surface chemistry and the luminescence is still under intense research. Experimental results and theoretical calculations showed that the luminescence of $\mathrm{SiC} \mathrm{NCs}$ is strongly influenced by the surface groups $^{15-17}$. While small SiC nanocrystals are often interpreted as a piece of bulk semiconductor ${ }^{18}$, recent studies ${ }^{15,16}$ imply that SiC NCs in aqueous environment may be described as complex macromolecules with formula $\mathrm{Si}_{x} \mathrm{C}_{y} \mathrm{O}_{z}(\mathrm{H})$. The surface modification of this complex system with a core of crystalline $\mathrm{SiC}$ can drastically affect its optical properties, as shown by previous theoretical calculations ${ }^{15}$. Ex- 
perimental results established that the optical properties of SiC NCs are also influenced by the environment and by intramolecular interactions ${ }^{12,15,16,19,20}$. However, the relatively broad size distribution and the possible distribution of surface terminators made it difficult to unambiguously prove the connection between surface termination and optical properties.

We apply the combination of advanced time resolved spectroscopic techniques and time-dependent density functional theory methods ${ }^{21}$, together with attenuated total reflectance infrared (ATR-IR) and steady-state photoluminescence (PL) spectroscopy on surface engineered colloid molecular-sized SiC NCs. Particularly, decay associated spectroscopy (DAS) and time-resolved area normalized emission spectroscopy (TRANES) methods, that have been so far applied only to few other systems ${ }^{22,23,24}$, substantially contribute to reveal the nature of the luminescence of SiC NCs. We applied time resolved emission spectroscopy (TRES) going beyond the conventional time-correlated single photon counting ${ }^{25-}$ ${ }^{28}$ that represents a collection of measurements using two independent variables: wavelength and the time after excitation. This results in a three-dimensional surface of data that allows to monitor the temporal evolution of the fluorescence as well as to resolve the spectrally overlapping species by using different analysis methods or models like TRANES and DAS. The strength of TRANES is that it reveals spectrally separated emitting centers independently of the shape of the individual decay functions ${ }^{29}$. While TRANES can identify the number of different emission centers in the solution, the DAS method can go beyond that and is able to reconstruct the steady state spectra of these centers if an appropriate spectral or temporal model is used ${ }^{30}$. We show here that DAS, which is widely used for organic fluorophores and biological species ${ }^{31}$ possessing a single exponential decay, can be applied to more complex systems such as semiconductor nanocrystals with non-exponential relaxation processes. DAS is able to identify a defect related recombination path with emission partly overlapping the main emission band from the nanocrystal. Our strategy is to simplify the complexity of the SiC NC surface by reduction and track the change in the optical properties of the resultant SiC NCs. We demonstrate successful identifi- 
cation of the surface and $\mathrm{pH}$ dependent luminescence of molecular-sized SiC NCs and unravel the presence of a silicon - oxygen bond related color center by reconstructing the decay associated spectra of SiC NCs. Our approach is useful for explanation of the fluorescence mechanism in other complex quantum dot and related systems.

\section{Results and discussion \\ Synthesis of different surface terminated SiC NCs}

Silicon carbide nanocrystals have been made by wet chemical etching method ${ }^{32}$. As-prepared SiC NCs of diameter between 1-4 nm were terminated with a variety of oxygen-containing species with high concentration of carboxyl groups ${ }^{15}$ that we label by 'as-prepared'. We prepared $-\mathrm{OH},-\mathrm{H}$ and $-\mathrm{SiO}_{\mathrm{x}}$ terminated SiC NCs by reduction of the as-prepared samples to simplify the surface termination of our NCs.. For the preparation of $-\mathrm{OH}$ terminated $\mathrm{SiC}$ NCs as-prepared $\mathrm{SiC}$ NCs were reduced by $\mathrm{NaBH}_{4}$ in aqueous solution (' $\mathrm{BH}_{4}{ }^{-}$reduced'). Hydrogen terminated $\mathrm{SiC}$ NCs were fabricated by reducing asprepared samples dispersed in $\mathrm{HCl}$ by dissolving $\mathrm{Zn}$ powder (' $\mathrm{Zn} / \mathrm{H}^{+}$reduced'). The oxidized sample ('reoxidized') was created by 2-hour illumination of the hydrogenated SiC NC sample with $320 \mathrm{~nm}$ wavelength light or a few days storage in water. Aging of $\mathrm{Zn} / \mathrm{H}^{+}$reduced SiC NCs was a little bit unexpected because of the known stability of $\mathrm{SiC}^{1}$. The conversion degree was studied by ATR-IR and steady-state PL spectroscopy. ATR-IR spectra of $\mathrm{Zn} / \mathrm{H}^{+}$reduced and reoxidized $\mathrm{SiC}$ NCs with size distribution of 5-30 nm are shown in Figure 1. This fraction of SiC NCs has the same surface properties as smaller (1-4 nm) $\mathrm{NCs}^{33}$ but can be purified from the reactant (see SI for more details). The broad peak at 900-1200 $\mathrm{cm}^{-1}$ attributed to the asymmetric stretching and bending modes of Si-O-Si, C-O-C, Si-O-C and $\mathrm{Si}-\mathrm{OH}$ bands is eliminated by the $\mathrm{Zn} / \mathrm{H}^{+}$reduction and is shifted to lower wavenumbers after reoxidation, confirming that the distribution and composition of these groups at the SiC NC surface is different. Vibrations of C-O-C and Si-O-C bonds usually appear at higher wavenumbers than those of Si-O-Si bonds ${ }^{34}$ while oxygen deficiency in $\mathrm{SiO}_{2}$ also shifts this type of vibration bands to lower wavenumbers $^{35}$. Reduction and reoxidation cause changes in the region of $3000-4000 \mathrm{~cm}^{-1}$, too. The as- 
prepared sample shows a strong and broad peak at around $3300 \mathrm{~cm}^{-1}$ because of the strongly hydrogenbonded $\mathrm{OH}$ groups on the surface and hydrogen-bonded water molecules ${ }^{15}$. Complete reduction removes the strong H-bonds, leaving only non-bonded $\mathrm{OH}$ groups behind (IR bands above $3600 \mathrm{~cm}^{-1}$ ). In the reoxidized sample the $\mathrm{OH}$ vibrational modes shift to lower wavenumbers and the $\mathrm{OH}$ band becomes broader, due to strongly $\mathrm{H}$-bonded $\mathrm{OH}$ groups partially formed on the developed oxide. In conclusion we found that after the $\mathrm{Zn} / \mathrm{H}^{+}$reduction most of the carbon and oxygen groups were eliminated from the surface and reoxidation enriched the surface with $\mathrm{Si}-\mathrm{O}$ bonds. ATR spectra for $\mathrm{OH}$ terminated $\mathrm{SiC}$ NCs can be found in the Supporting Information (SI).

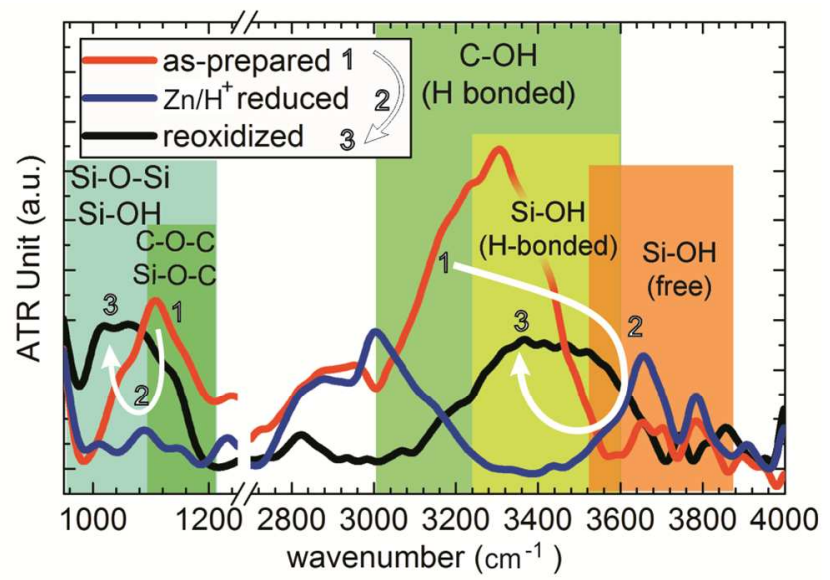

Figure 1.: ATR-IR spectra of as prepared, $\mathrm{Zn} / \mathrm{H}+$ reduced and reoxidized SiC NCs. Reduction eliminates most of the $\mathrm{C}-\mathrm{O}$ bonds while during reoxidation $\mathrm{Si}-\mathrm{OH}$ and $\mathrm{Si}-\mathrm{O}-\mathrm{Si}$ bonds form.

\section{Steady state photoluminescence study of different surface terminated SiC NCs}

The effect of the reduction processes was monitored by steady state PL measurements, too. Figure 2 shows the PL spectra of the samples at $320 \mathrm{~nm}$ excitation. There is a clear blue shift with increasing reduction degree with emission maximum at 450, 435 and $380 \mathrm{~nm}$ for as-prepared, $\mathrm{BH}_{4}{ }^{-}$reduced and $\mathrm{Zn} / \mathrm{H}^{+}$reduced samples, respectively. While the as-prepared and $\mathrm{OH}$ terminated $\left(\mathrm{BH}_{4}\right.$ reduced) samples show no changes in the emission spectra at the surveillance time, the emission of $\mathrm{Zn} / \mathrm{H}^{+}$sample shows 
significant time evolution by shifting the peak maximum from around $380 \mathrm{~nm}$ to $410 \mathrm{~nm}$ during the reoxidation process of 2 hours.

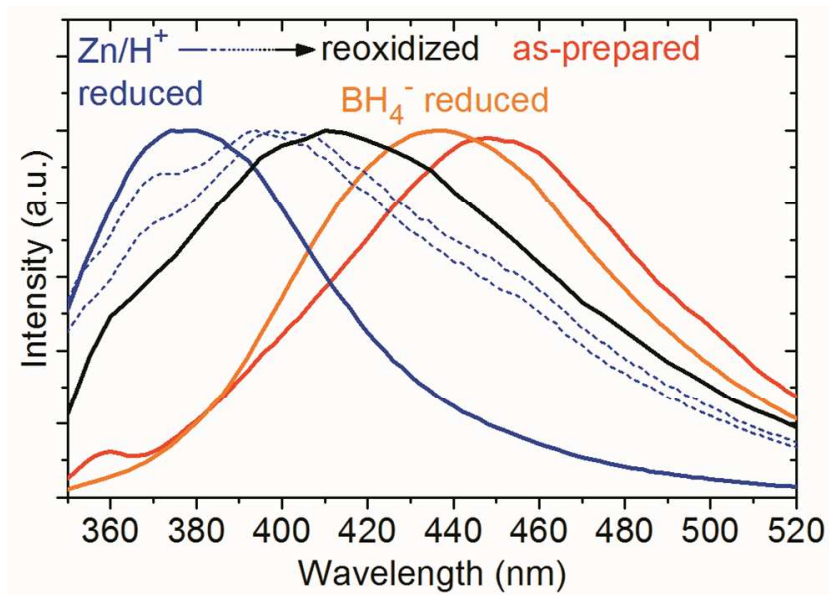

Figure 2.: Steady state PL spectra of different surface terminated SiC NCs. Excitation wavelength is 320 $\mathrm{nm}$. The emission maxima are at 455, 435, 380 and $410 \mathrm{~nm}$ for as-prepared, BH4- reduced, $\mathrm{Zn} / \mathrm{H}+\mathrm{re}-$ duced and reoxidized samples, respectively. Blue dotted lines represent the emission upon oxidation of the $\mathrm{Zn} / \mathrm{H}+$ reduced sample as a function of illumination time from left to right. The final spectral shape is reached after 2-hour illumination (black line) using $320 \mathrm{~nm}$ excitation.

\section{Wavelength dependent time-resolved emission spectroscopy studies}

Wavelength dependent time-resolved emission measurements were carried out at $321 \mathrm{~nm}$ excitation in all samples. Emission is measured in the 340-570 nm wavelength range with $10 \mathrm{~nm}$ steps (see SI for more details). Decay curves free from excitation pulses' distortion were reconstructed using deconvolution and then were used for TRANES and DAS analysis.

\section{Time resolved area normalized emission spectroscopy studies}

TRANES is a model-free method which indicates the number of emission centers in the monitored system $^{29}$. We found that the time-dependent spectra in case of every sample form a single isoemissive point that implies definitely two emission centers in our samples (figure 3.). This is intriguing because Kasha's rule implies non-radiative recombination from the higher-level excited states toward the lowest energy excited state. If the higher energy excited states associated with the radiative decay are related to 
a surface group as expected from the steady state PL spectra and from theoretical calculations, changing the surface chemistry should change the lowest energy states and an isoemissive point should not be found. If the emission is due to the recombination on different localized surface states then a decreasing number of isoemissive points should be observed by increasing the surface reduction degree. The presence of two emission centers in all type of samples cannot be described with the usual relaxation theory. While we can identify two separate centers with TRANES, this method is unable to reconstruct the spectra of the detected emission centers. To this end, we also applied DAS.

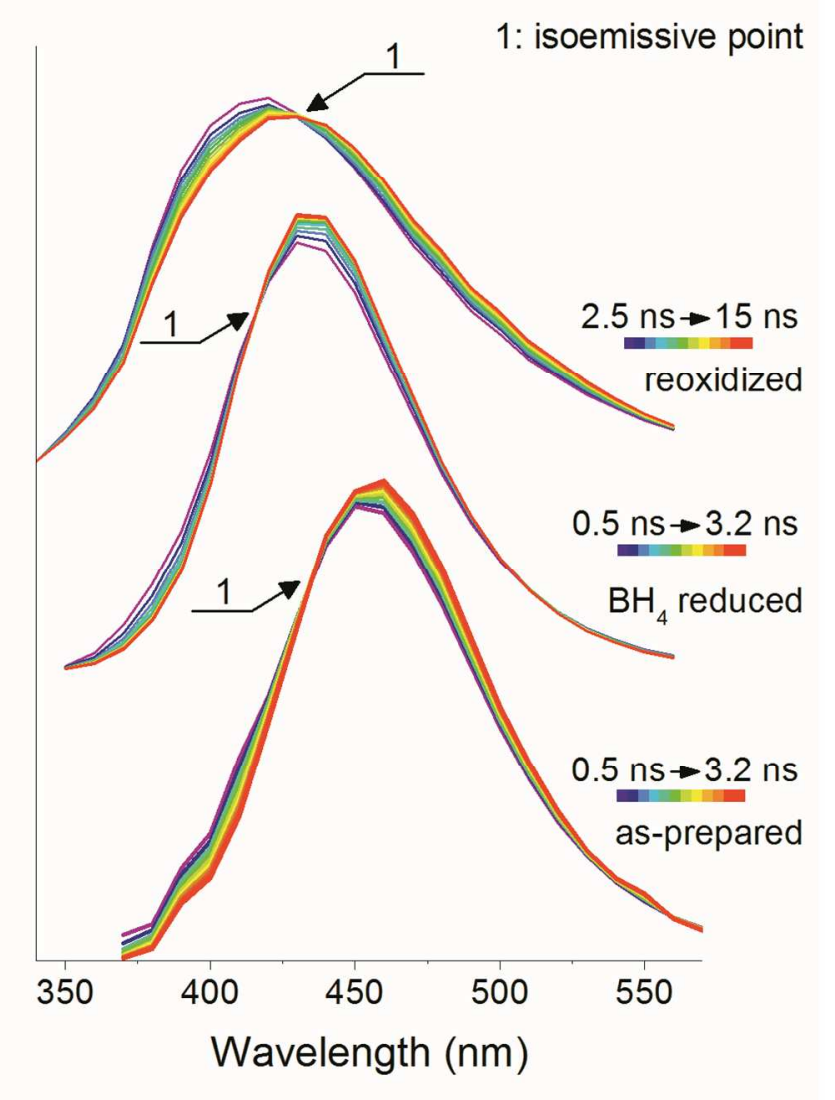

Figure 3. TRANES curves of the as-prepared, BH4 reduced and the reoxidized form $\mathrm{Zn} / \mathrm{H}+$ reduced samples. The y-axis is the fluorescence intensity in arbitrary unit. The zero levels vary from sample to sample. This way of representation was chosen for the sake of visibility. Notice that the plotted time intervals are $0.5 \mathrm{~ns}$ to $3.2 \mathrm{~ns}$ for the as-prepared and for the BH4 reduced samples, while the plotted pe- 
riod is $2.5 \mathrm{~ns}$ to $15 \mathrm{~ns}$ for the reoxidized from $\mathrm{Zn} / \mathrm{H}+$ reduced sample. In the case of the reoxidized sample the shorter wavelength and shorter lifetime emission center gives more intensive emission, thus TRANES yields the isoemissive point on a longer time scale. Figure S6 shows the DAS for the three samples.

\section{Decay associated spectra analysis.}

The DAS analysis method yields the individual spectra of the mixed fluorophores and the summation of these spectra reproduces the steady state PL. In order to fit the decay curves for DAS analysis of the three samples, 5-exponential fit was applied in all cases. For DAS analysis fixed time constants have to be used in the global analysis. These 5 exponentials do not directly imply at all that 5 individual emission centers occur in our samples. These decay components are just a result of an excellent fit for nonmonoexponential decay with chi-square of 1.0-1.5 at every wavelength. SiC NCs have a relatively large distribution of size and surface terminators which cause non-exponential decay and time dependent spectral shift in TRES. As a result, spectra and lifetimes given from DAS analysis have no exact physical meaning. Nevertheless, by analyzing the shape and maxima of DAS, it is possible to draw some conlcusions $^{36}$. By taking into account the variation of the emission wavelengths of a single type luminescent center in the model of the DAS method, DAS rather shows two different bands in all the three samples. From DAS analysis using 5-exponential fit the as-prepared sample shows 3 broad features with maximum at $450-460 \mathrm{~nm}$ and 2 peaks at $410-420 \mathrm{~nm}$. The $\mathrm{NaBH}_{4}$ reduced sample has 4 peaks at $\sim 435$ $\mathrm{nm}$ with different lifetimes and one peak at $410-420 \mathrm{~nm}$. In the case of the reoxidized sample the DAS fit gives 4 peaks with 410-420 nm peak maximum and one at $435 \mathrm{~nm}$. Figure 4 shows the reconstructed sub-spectra of the three samples. Curves with different lifetimes but same maxima are weighted together and this representation gives a very clear visualization of two different emission centers. 


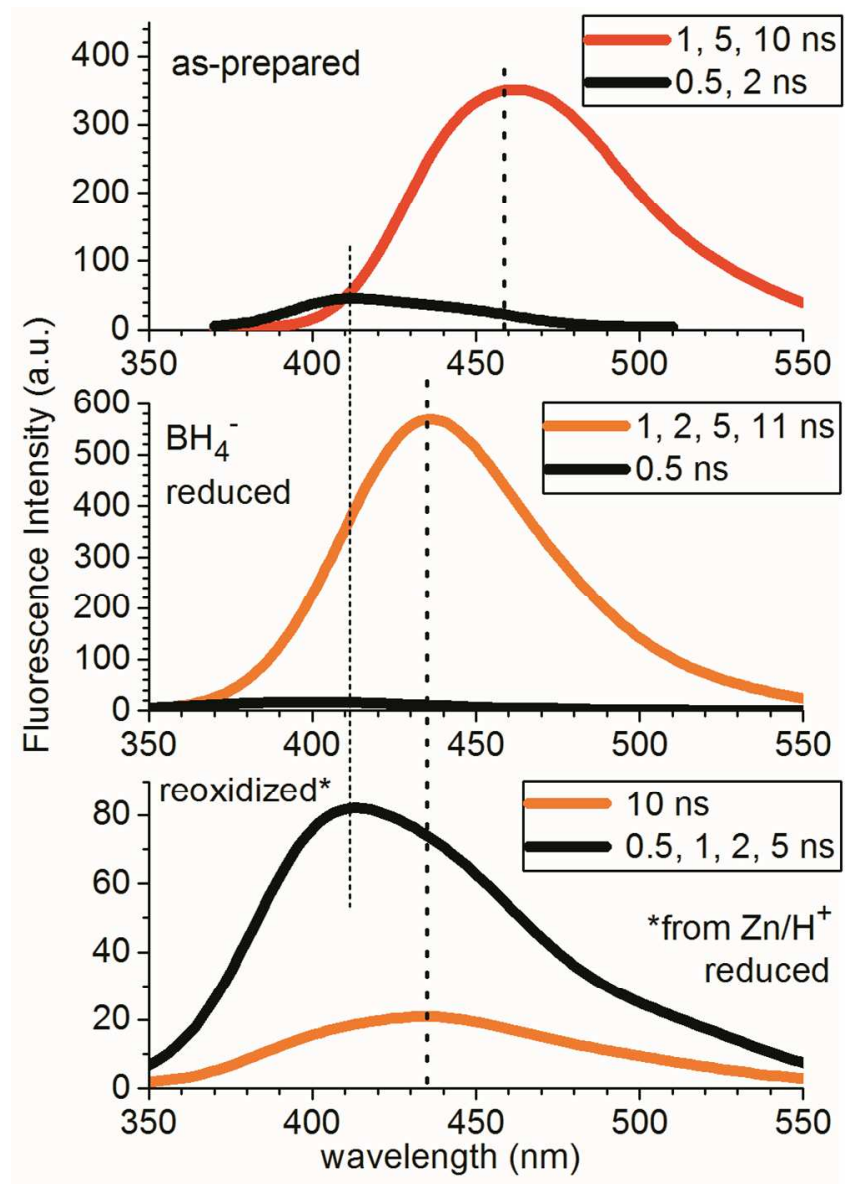

Figure 4.: Decay associated spectra (DAS) of as-prepared and reduced samples. Spectra having different lifetime components but the same maxima are weighted together for the sake of visibility. DAS confirms two emission centers in SiC NCs. The emission at $\sim 410 \mathrm{~nm}$ is due to the presence of fluorescent defects on the oxidized surface.

In consistence with the result of TRANES analysis, DAS also indicates two luminescence centers in all samples and by reconstructing the two emission spectra we are able to explain the presence of two types of fluorescence centers in single $\mathrm{SiC}$ NC systems. DAS indicate that one of the found emission centers does not change during the chemical modifications and has a short lifetime in all samples. The other one shifts with chemical modification. Combining this result with the ATR-IR spectra and $a b$ initio theory we conclude that a common emitter forms in all the samples with wavelengths at 410-420 nm which may be associated with some defects on the oxidized SiC NC surface. Indeed, silicon dioxide 
$\left(\mathrm{SiO}_{2}\right)$ has numerous luminescent defects emitting at these wavelengths ${ }^{37}$ and our theoretical calculations prove that surface groups tailor the absorption band of the nanocrystal instead of forming individual localized emission centers, while $\mathrm{Si}$ - O defects create localized states and have "individual" absorption and emission peaks ${ }^{38}$. These $\mathrm{Si}-\mathrm{O}$ defect related emitters give little contribution to the overall emission in the as-prepared samples because $\mathrm{SiO}_{2}$-like oxide is not the main compound on the surface, and even smaller in $\mathrm{BH}_{4}{ }^{-}$reduced samples where the oxygen bridges were slightly eliminated. However, they become dominant in reoxidized samples where carbon-oxygen groups were eliminated by the $\mathrm{Zn} / \mathrm{H}^{+}$reduction, the highly reduced surface reconstructed in water forming $\mathrm{Si}-\mathrm{O}$ bonds on the fresh $\mathrm{Si}$ surfaces of $\mathrm{SiC} \mathrm{NCs,} \mathrm{and} \mathrm{some} \mathrm{C}-\mathrm{OH}$ groups also formed according to the ATR-IR spectra.

Analysis of the time resolved spectra reveals $\mathrm{Si}-\mathrm{O}$ defect related color centers that are hidden in the steady-state PL spectra. In addition, the difference in the emission of as-prepared and $\mathrm{BH}_{4}{ }^{-}$reduced samples is also observable as a $\sim 20 \mathrm{~nm}$ blue shift upon reduction which follows Kasha's rule indicating that changing the surface chemistry changes the highest energy states of the nanocrystal as we expected from steady state PL spectra and from the results of $a b$ initio calculations.

\section{Titrimetric investigation of surface groups}

The emission of SiC NCs shows $\mathrm{pH}$ dependency which was associated with surface changes, especially with dissociation of carboxyl groups followed by changes of intramolecular hydrogen bonds ${ }^{16}$ or with the presence of $\mathrm{Si}-\mathrm{OH}$ bonds on the surface ${ }^{39}$, but from the results of quantum mechanical calculations it was concluded that $\mathrm{SiC} \mathrm{NCs} \mathrm{in} \mathrm{aqueous} \mathrm{solutions} \mathrm{are} \mathrm{not} \mathrm{sensitive} \mathrm{to} \mathrm{the} \mathrm{dissociation} \mathrm{of} \mathrm{car-}$ boxyl groups. We investigate this effect and further corroborate our model with titrimetric studies of oxygenated groups of SiC NCs. We found numerous oxides on the surface of SiC NCs. Most of the oxygenated carbon and silicon groups have acid-base characteristics. We measured the relative quantity and contribution to the luminescence of these groups in order to follow the photoacidic characteristics of SiC NCs. We performed potentiometric titration from $\mathrm{pH} 2$ to $\mathrm{pH} 13$ and monitored the PL signals. We 
found three dissociation processes with $\mathrm{pK}_{\mathrm{a}} 4.2,7.1$, and 10.0. The $\mathrm{pK}_{\mathrm{a}} 4.2$ is attributed to the dissociation of carboxyl groups and $\mathrm{pK}_{\mathrm{a}} 10$ is due to the dissociation of hydroxyl groups. $\mathrm{pK}_{\mathrm{a}} 7.1$ could be the dissociation of sylanol groups. These are the three major dissociative groups that usually occur on the surface of SiC NCs. By tracking the dissociation with PL we found that the peak at $450 \mathrm{~nm}$ shifts to 435 $\mathrm{nm}$ at around $\mathrm{pH} 9$ which does not match the measured inflexion points. In the case of back titration of the basic samples the emission maximum shifts back to $450 \mathrm{~nm}$ at about $\mathrm{pH} 4$ that implies a hysteresis loop in the PL by changing the $\mathrm{pH}$ value. As a consequence, the emission shift during titration cannot be associated with simple dissociation of a given surface group. It could be due to quenching effect of alkali ions which form complexes with dissociated carboxyl groups at high concentration. To clarify this, we increase the ionic strength with addition of $\mathrm{NaCl}$ at $\mathrm{pH} 7$ where carboxyl groups are dissociated but the PL remains unchanged. Indeed, the PL is shifted to $435 \mathrm{~nm}$ after this treatment proving that the blue shift is not associated only with the dissociation of surface groups. According to our calculations, carboxyl groups shift the highest occupied molecular orbital (HOMO) level associated with surface $\mathrm{C}-\mathrm{OH}$ groups. When a non-hydrogen cation interacts with carboxyl groups this energy shift decreases and the HOMO level becomes similar to that of $\mathrm{OH}$ terminated SiC NCs's. The titration curve with marked color changes is depicted in Figure 5.

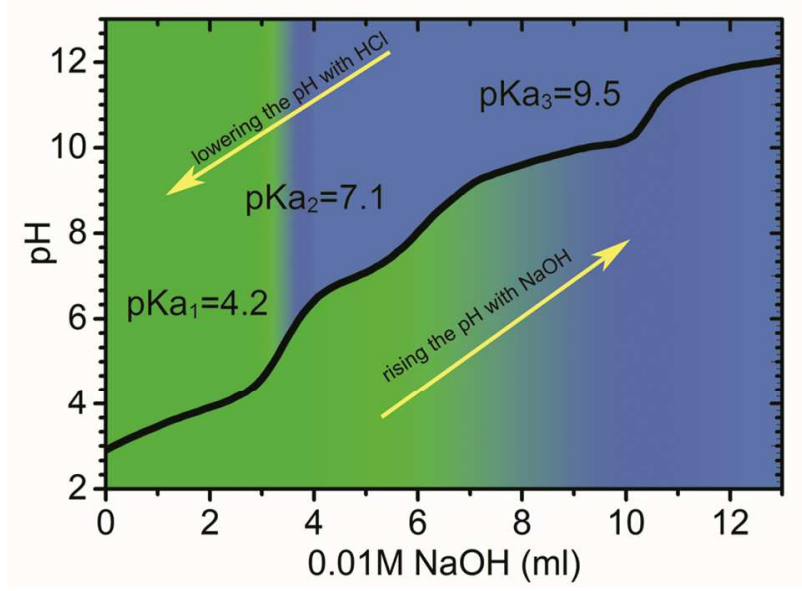


Figure 5.: Titration curve of SiC NCs in the 2-13 pH range. Color changes below the titration curve represent the color changes of the solution when $\mathrm{pH}$ was changed from 2 to 13 . Color changes above the curve represent the color changes of the solution when $\mathrm{pH}$ was changed from 13 to 2.

Our results imply that either substitution of carboxyl groups by hydroxyl groups or quenching them by alkenes causes about the same blue shift in PL. Different solvatochromic shifts were also reported $^{19,40}$ demonstrating the surface sensitivity of the obtained PL, but much larger size distribution of $\mathrm{SiC}$ particles was applied in those studies that can seriously alter the spectrum with respect to molecular-sized SiC NCs. ${ }^{33}$

\section{Ab initio calculations}

These experimental results are supported by our time-dependent density functional theory (TDDFT) results on the calculated excitation spectrum of carboxyl and hydroxyl terminated surfaces that we already applied successfully in the context. ${ }^{15,17,21}$ The details about the methodology are given in the SI.

We have chosen a small but realistic $\mathrm{SiC}$ nanocrystal with diameter of $1.4 \mathrm{~nm}$ as a basic model in our calculations. Initially the dangling bonds on the surface were terminated by hydrogen atoms, with the chemical formula of $\mathrm{Si}_{79} \mathrm{C}_{68} \mathrm{H}_{100}$. SiC nanocrystals of this size are experimentally relevant and small enough to allow cost-effective simulations.

We substituted the hydrogen atoms on the surface with various chemical groups such as $\mathrm{Si}-\mathrm{OH}, \mathrm{C}$ $\mathrm{OH}$, Si-COOH, C-COOH and Si-O-Si bridges. We considered the following different models: $i$ ) hydrogen atoms substituted with -OH groups and $\mathrm{Si}-\mathrm{O}-\mathrm{Si}$ groups on the silicon-terminated faces of the nanocrystal; ii) hydrogen atoms substituted with -OH groups on both the silicon- and carbon-terminated faces of the nanocrystal; iii) majority of the hydrogen atoms substituted with -OH groups on both the silicon- and carbon-terminated faces of the nanocrystal, the remaining hydrogen atoms substituted with $\mathrm{COOH}$ moieties; (iiia and iiib refers to carboxyl groups on the carbon and silicon terminated surfaces of 
the nanocrystals, respectively) iv) majority of the hydrogen atoms substituted with -OH groups on both the silicon- and carbon-terminated faces of the nanocrystal, the remaining hydrogen atoms substituted with $-\mathrm{COO}^{-} \mathrm{Na}^{+}$moieties; v) hydrogen atoms substituted with -OH groups on the silicon terminated surface while the carbon atoms on the originally carbon terminated surface were substituted with oxygen atoms to form a thin $\mathrm{SiO}_{2}$ layer.

The calculated optical gaps are summarized in Table S2 and Figure S7 of the SI. It is important to note that these TDDFT results concern the optical absorption of the model SiC nanocrystals, thus straightforward comparison to the PL emission spectra is not possible. Nevertheless, the effect of the Stokes shift is unlikely to alter the calculated trend of the optical gaps as a function of the surface treatment. The calculated change in the optical gaps is in qualitative agreement with the PL data. The "asprepared" sample has the lowest optical gap which increases as the carboxyl groups are removed from the surface ("BH 4 reduced") and further increases as both the carboxyl and $\mathrm{C}-\mathrm{OH}$ groups are reduced (“ $\mathrm{Zn} / \mathrm{H}^{+}$reduced"). With the addition of $\mathrm{NaCl}$, the carboxyl groups dissociate, and the positive sodium ions bind to the negative carboxyl ion. In this case, the calculated optical gap shows a blue-shift which is in accordance with the measurements. The oxidation of the carbon-terminated surface leads to a thin $\mathrm{SiO}_{2}$ layer on the surface of the $\mathrm{SiC}$ nanocrystal. This model has the largest optical gap according to our calculations. While the blue-shift compared to the "as-prepared" sample is also observed in the experiments, the calculations predict that the "reoxidized" sample has the lowest wavelength emission. However, the emission in the reoxidized samples comes from defects in the surface $\mathrm{SiO}_{2}$ matrix or the $\mathrm{SiC} / \mathrm{SiO}_{2}$ interface. The corresponding defects were not yet identified in the $\mathrm{SiO}_{2}$ matrix. The identification of these color centers exceeds the scope of this work.

To understand the effect of the surface on the optical properties, we analyzed the density of states (DOS). While the DOS does not take into account the excitonic effects, it provides information about 
the electronic structure. Figure 6 shows the calculated projected density of states (PDOS) for SiC NCs with different surface terminations. Apparently, the substitution of hydrogen in Si-H bonds by hydroxyl groups does not change the density of states of the $\mathrm{SiC}$ nanocrystal significantly. On the other hand, when the hydrogens in $\mathrm{C}-\mathrm{H}$ bonds are also substituted by hydroxyl groups the density of states changes as surface states appear near the valence band edge that are localized to the C-OH surface. Carboxyl moieties on the Si-terminated surface cause further changes in the density of states: while the states near the valence band edge remain localized on the $\mathrm{C}-\mathrm{OH}$ surfaces, the band gap decreases. On the contrary, carboxyl groups on the C-terminated surfaces of the $\mathrm{SiC}$ nanocrystal have little to no effect on the density of states. As the carboxyl groups on the Si surfaces are substituted with -COO-Na+ groups, the optical gap increases by a small amount. The reoxidized sample possesses the largest band gap and absorption edge which may be associated with two effects: (i) the elimination of C-OH groups from the surface and (ii) the reduction of the core size of the $\mathrm{SiC}$ nanocrystal (quantum confinement effect). Comparing the DAS reconstructed luminescence of SiC NCs and the experimental data in silicon dioxide ${ }^{37}$ indicates that the luminescence can be tentatively associated with the so-called weak-oxygen-bond defect in the oxide shell. Unambiguous identification of the color center in the oxide shell layer of SiC NC is beyond the scope of this study as this defect has not yet been identified since decades in bulk silicon dioxide. 


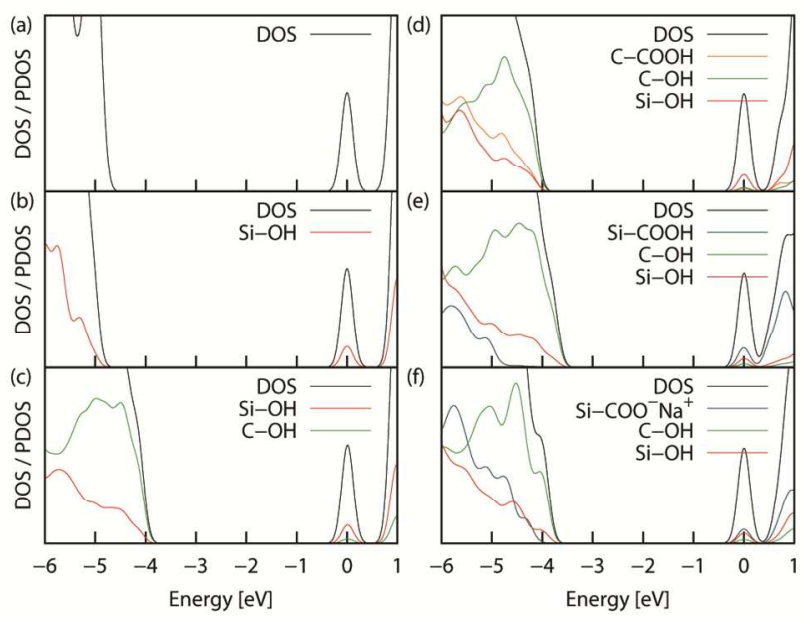

Figure 6.: Density of states (DOS) and projected density of states (PDOS) of our model SiC nanocrystal with different surface terminations: (a) hydrogenated surface, (b) " $\mathrm{Zn} / \mathrm{H}+$ " reduced and (c) BH4 reduced. (d, e) DOS/PDOS of the model related to the "as prepared" sample, where the carboxyl groups bond to the C-and Si-terminated surface of the nanocrystals, respectively. (f) DOS/PDOS for the model of the sample with added $\mathrm{NaCl}$ where the carboxyl groups are dissociated. For the sake of easier comparison, the DOS/PDOS was shifted along the energy axis until the peak associated with the lowest unoccupied molecular orbital is at $0 \mathrm{eV}$. The DOS and PDOS were calculated with the PBE0 exchangecorrelation functional and double- $\zeta$ polarized basis set.

\section{Conclusions}

The complexity of as-prepared SiC NCs results in a subtle PL mechanism. We successfully prepared hydroxyl and silicon oxide terminated SiC NCs using different surface reduction methods and we demonstrated that elimination of both carboxyl and hydroxyl groups by reduction causes a dramatic blue shift in PL. Wavelength dependent time-resolved luminescence spectroscopy was used to study the luminescence properties of SiC NCs with different surface terminations. We successfully applied DAS reconstruction of the steady-state spectra from the decay curves in a highly disperse system and we identified $\mathrm{SiO}_{x}$ defect related color centers at the surface of SiC NCs. From the experimental data we built a framework for the surface related luminescence which can describe both the environment sensi- 
tivity and the connection between luminescence and surface chemistry. We summarize the connection between surface chemistry and the PL properties of SiC NCs in Scheme 1.

Scheme 1. Surface and environment dependent luminescence of SiC NCs

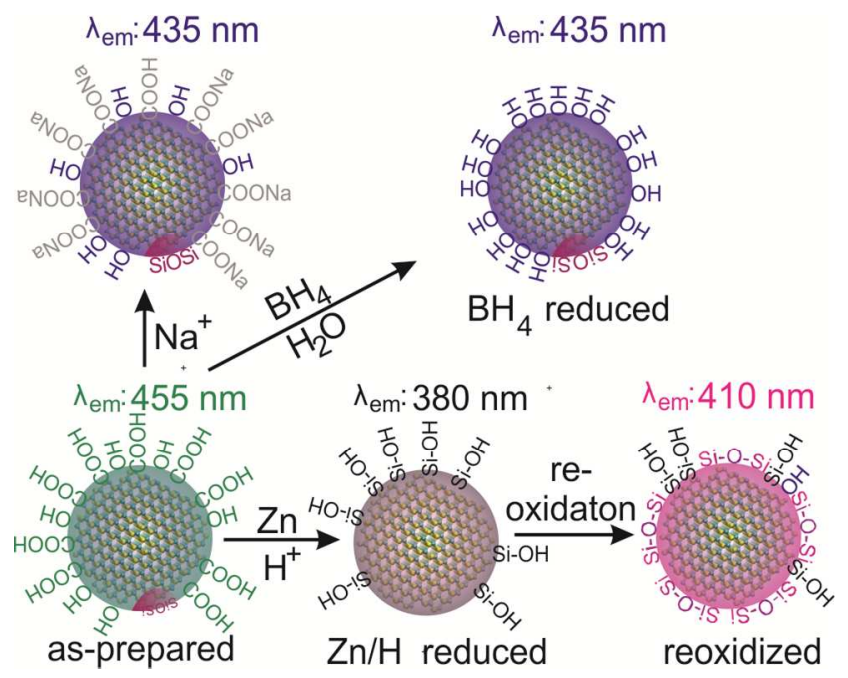

Our results imply that the color centers introduced in the core of SiC $\mathrm{NCs}^{41,42}$ might interfere with the surface groups, thus careful surface engineering is inevitable in the fabrication of SiC NC based nanosensors.

\section{ASSOCIATED CONTENT}

Supporting Information. Surface modification reactions of SiC NCs, ATR-IR measurement description, PL and time resolved PL measurement description, titration experiment details, details of calculation. This material is available free of charge via the Internet at http://pubs.acs.org.

\section{AUTHOR INFORMATION}

\section{Corresponding Authors}

* Adam Gali: gali.adam@wigner.mta.hu, David Beke: beke.david@wigner.mta.hu

\section{Author Contributions}

D.B conceived the idea of this project, fabricated the material, carried out IR and PL measurements, participated in the analysis of the TRES related results and the titration studies. T.Z.J and J.E carried out the TRES related measurements and participated in 
the analysis of the data. Zs.Sz and K.K carried out IR measurements and interpreted the IR spectra. D.Á.M contributed to the titration studies and analysis. A.G supervised the project and wrote the paper. The manuscript was written through contributions of all authors.

\section{ACKNOWLEDGMENT}

Zs.Sz and K.K acknowledge the joint project of the Scientific Research fund (OTKA) and the Austrian Science Fund (FWF) under Grant No. ANN107580. D.B acknowledges the support from the European Union and the State of Hungary, co-financed by the European Social Fund in the framework of TÁMOP-4.2.4.A/2-111/1-2012-00001 National Excellence Program. A.G acknowledges the support from the Hungarian Scientific Fund (OTKA) project nos. K101819 and K106114, and the Lendület program of Hungarian Academy of Sciences. J.T.Z and E.J acknowledge the grant SROP-4.2.2.D15/1/Konv-2015-0015: Environmental industry related innovative trans- and interdisciplinary research team development in the University of Pécs knowledge base.

\section{REFERENCES}

(1) Selmane Bel Hadj Hmida, E.; Properties and applications of silicon carbide; Rosario, G., Ed.; InTech, 2011.

(2) Palmour, J. W.; Edmond, J. A.; Kong, H. S.; Carter Jr., C. H. Phys. B Condens. Matter 1993, $185(1-4), 461$.

(3) Koehl, W. F.; Buckley, B. B.; Heremans, F. J.; Calusine, G.; Awschalom, D. D. Nature 2011, $479(7371), 84$.

(4) Falk, A. L.; Buckley, B. B.; Calusine, G.; Koehl, W. F.; Dobrovitski, V. V; Politi, A.; Zorman, C. A.; Feng, P. X.-L.; Awschalom, D. D. Nat. Commun. 2013, 4 (May), 1819.

(5) Castelletto, S.; Johnson, B. C.; Ivády, V.; Stavrias, N.; Umeda, T.; Gali, A.; Ohshima, T. Nat. Mater. 2013, $12(11), 1$. 
(6) Widmann, M.; Lee, S.-Y.; Rendler, T.; Son, N. T.; Fedder, H.; Paik, S.; Yang, L.-P.; Zhao, N.;

Yang, S.; Booker, I.; Denisenko, A.; Jamali, M.; Momenzadeh, S. A.; Gerhardt, I.; Ohshima, T.; Gali, A.; Janzén, E.; Wrachtrup, J. Nat. Mater. 2015, 14, 164.

(7) Fuchs, F.; Soltamov, V. A.; Väth, S.; Baranov, P. G.; Mokhov, E. N.; Astakhov, G. V; Dyakonov, V. Sci. Rep. 2013, 3, 1637.

(8) Barillet, S.; Jugan, M. L.; Laye, M.; Leconte, Y.; Herlin-Boime, N.; Reynaud, C.; Carrière, M. Toxicol. Lett. 2010, 198 (3), 324.

(9) Beke, D.; Szekrényes, Zs.; Pálfi, D.; Róna, G.; Balogh, I.; Maák, P. A.; Katona, G.; Czigány, Z.; Kamarás, K.; Rózsa, B.; Buday, L.; Vértessy, B.; Gali, A. J. Mater. Res. 2013, 28 (02), 205.

(10) Saddow, S. E.; Locke, C. W.; Severino, A.; La Via, F.; Reyes, M.; Register, J.; Saddow, S. E. Silicon Carbide Biotechnology, First Edit.; Elsevier Inc., 2012.

(11) Fan, J. Y.; Wu, X. L.; Zhao, P. Q.; Chu, P. K. Phys. Lett. Sect. A Gen. At. Solid State Phys. 2006, $360(2), 336$.

(12) Beke, D.; Szekrényes, Zs.; Balogh, I.; Veres, M.; Fazakas, É.; Varga, L. K.; Kamarás, K.; Czigány, Z.; Gali, A. Appl. Phys. Lett. 2011, 99 (21), 213108.

(13) Botsoa, J.; Lysenko, V.; Géloën, A.; Marty, O.; Bluet, J. M.; Guillot, G. Appl. Phys. Lett. 2008, $92(17), 173902$.

(14) Mognetti, B.; Barberis, A.; Marino, S.; Di Carlo, F.; Lysenko, V.; Marty, O.; Géloën, A. J. Nanosci. Nanotechnol. 2010, 10 (12), 7971.

(15) Szekrényes, Zs.; Somogyi, B.; Beke, D.; Károlyházy, G.; Balogh, I.; Kamarás, K.; Gali, A. J. Phys. Chem. C 2014, 118 (34), 19995. 
(16) Dai, D.; Guo, X.; Fan, J. Appl. Phys. Lett. 2015, 106 (5), 053115.

(17) Vörös, M.; Deák, P.; Frauenheim, T.; Gali, A. J. Chem. Phys. 2010, 133 (6), 064705.

(18) Wu, X. L.; Fan, J. Y.; Qiu, T.; Yang, X.; Siu, G. G.; Chu, P. K. Phys. Rev. Lett. 2005, 94 (2), 026102 .

(19) Fan, J. Y.; Wu, X. L.; Li, H. X.; Liu, H. W.; Siu, G. G.; Chu, P. K. Appl. Phys. Lett. 2006, 88 (4), 041909.

(20) Fan, J. Y.; Li, H. X.; Cui, W. N.; Dai, D. J.; Chu, P. K. Appl. Phys. Lett. 2010, 97 (19), 191911.

(21) Somogyi, B.; Gali, A. J. Phys. Condens. Matter 2014, 26 (14), 143202.

(22) Lakowicz, J. R.; Gryczynski, I.; Gryczynski, Z.; Nowaczyk, K.; Murphy, C. J. Anal. Biochem. $\mathbf{2 0 0 0 ,} 280(1), 128$.

(23) Konkena, B.; Vasudevan, S. J. Phys. Chem. Lett. 2014, 5 (1), 1.

(24) Tiseanu, C.; Kumke, M. U.; Parvulescu, V. I.; Koti, A. S. R.; Gagea, B. C.; Martens, J. A. J. Photochem. Photobiol. A Chem. 2007, 187 (2-3), 299.

(25) Van Driel, A. F.; Allan, G.; Delerue, C.; Lodahl, P.; Vos, W. L.; Vanmaekelbergh, D. Phys. Rev. Lett. 2005, 95 (23), 236804.

(26) Berstermann, T.; Auer, T.; Kurtze, H.; Schwab, M.; Yakovlev, D. R.; Bayer, M.; Wiersig, J.; Gies, C.; Jahnke, F.; Reuter, D.; Wieck, a. D. Phys. Rev. B - Condens. Matter Mater. Phys. 2007, 76 (16), 165318.

(27) Van Driel, A. F.; Nikolaev, I. S.; Vergeer, P.; Lodahl, P.; Vanmaekelbergh, D.; Vos, W. L. Phys. Rev. B - Condens. Matter Mater. Phys. 2007, 75 (3), 035329. 
(28) Nikolaev, I. S.; Lodahl, P.; Van Driel, A. F.; Koenderink, A. F.; Vos, W. L. Phys. Rev. B Condens. Matter Mater. Phys. 2007, 75 (11), 115302.

(29) Koti, A. S. R.; Periasamy, N. J. Chem. Phys. 2001, 115 (15), 7094.

(30) Loefroth, J. E. J. Phys. Chem. 1986, 90 (6), 1160.

(31) Kumpulainen, T.; Brouwer, A. M. Phys. Chem. Chem. Phys. 2012, 14 (37), 13019.

(32) Beke, D.; Szekrényes, Z.; Balogh, I.; Czigány, Z.; Kamarás, K.; Gali, A. J. Mater. Res. 2013, 28 (01), 44 .

(33) Beke, D.; Szekrényes, Z.; Czigány, Z.; Kamarás, K.; Gali, Á. Nanoscale 2015, 7 (25), 10982.

(34) Jing, S.; Lee, H. J. Korean Phys. Soc. 2002, 41 (5), 769.

(35) Tomozeiu, N. In Optoelectronics Materials and Techniques; Prof. P. Predeep, Ed.; InTech, 2011; pp 56-101.

(36) Marciniak, H.; Lochbrunner, S. Chem. Phys. Lett. 2014, 609, 184.

(37) Cheang-Wong, J. C.; Oliver, A.; Roiz, J.; Hernández, J. M.; Rodríguez-Fernández, L.; Morales, J. G.; Crespo-Sosa, A. In Nuclear Instruments and Methods in Physics Research, Section B: Beam Interactions with Materials and Atoms; 2001; Vol. 175-177, pp 490-494.

(38) Vörös, M.; Gali, A.; Kaxiras, E.; Frauenheim, T.; Knaup, J. M. Phys. Status Solidi Basic Res. 2012, 249 (2), 360.

(39) Wu, X. L.; Xiong, S. J.; Zhu, J.; Wang, J.; Shen, J. C.; Chu, P. K. Nano Lett. 2009, 9 (12), 4053.

(40) Zakharko, Y.; Botsoa, J.; Alekseev, S.; Lysenko, V.; Bluet, J.-M.; Marty, O.; Skryshevsky, V. A.; Guillot, G. J. Appl. Phys. 2010, 107 (1), 013503. 
21

23

24

26

27

28

30

31

33

34

35

36

37

39

40

41

42

44

45

46

47

48

49

51

52

53

54

55

57

58

59

60 


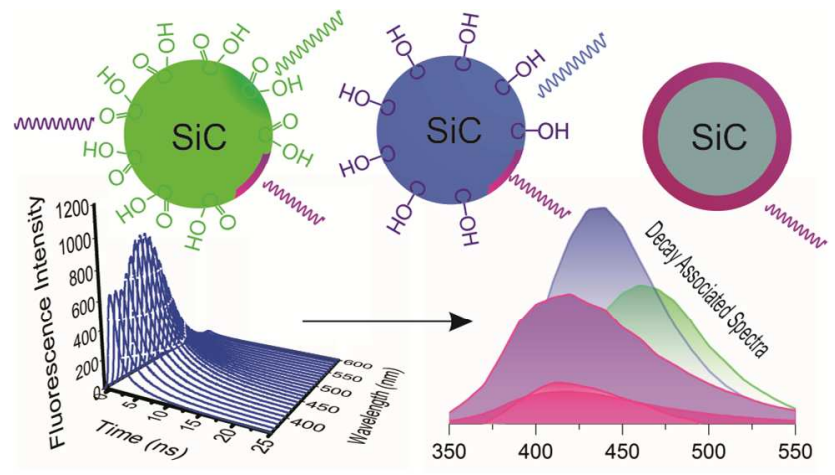


Figure 1.: ATR-IR spectra of as prepared, $\mathrm{Zn} / \mathrm{H}+$ reduced and reoxidized SiC NCs. Reduction elimi-nates most of the $\mathrm{C}-\mathrm{O}$ bonds while during reoxidation $\mathrm{Si}-\mathrm{OH}$ and $\mathrm{Si}-\mathrm{O}-\mathrm{Si}$ bonds form. $85 \times 60 \mathrm{~mm}(300 \times 300$ DPI) 


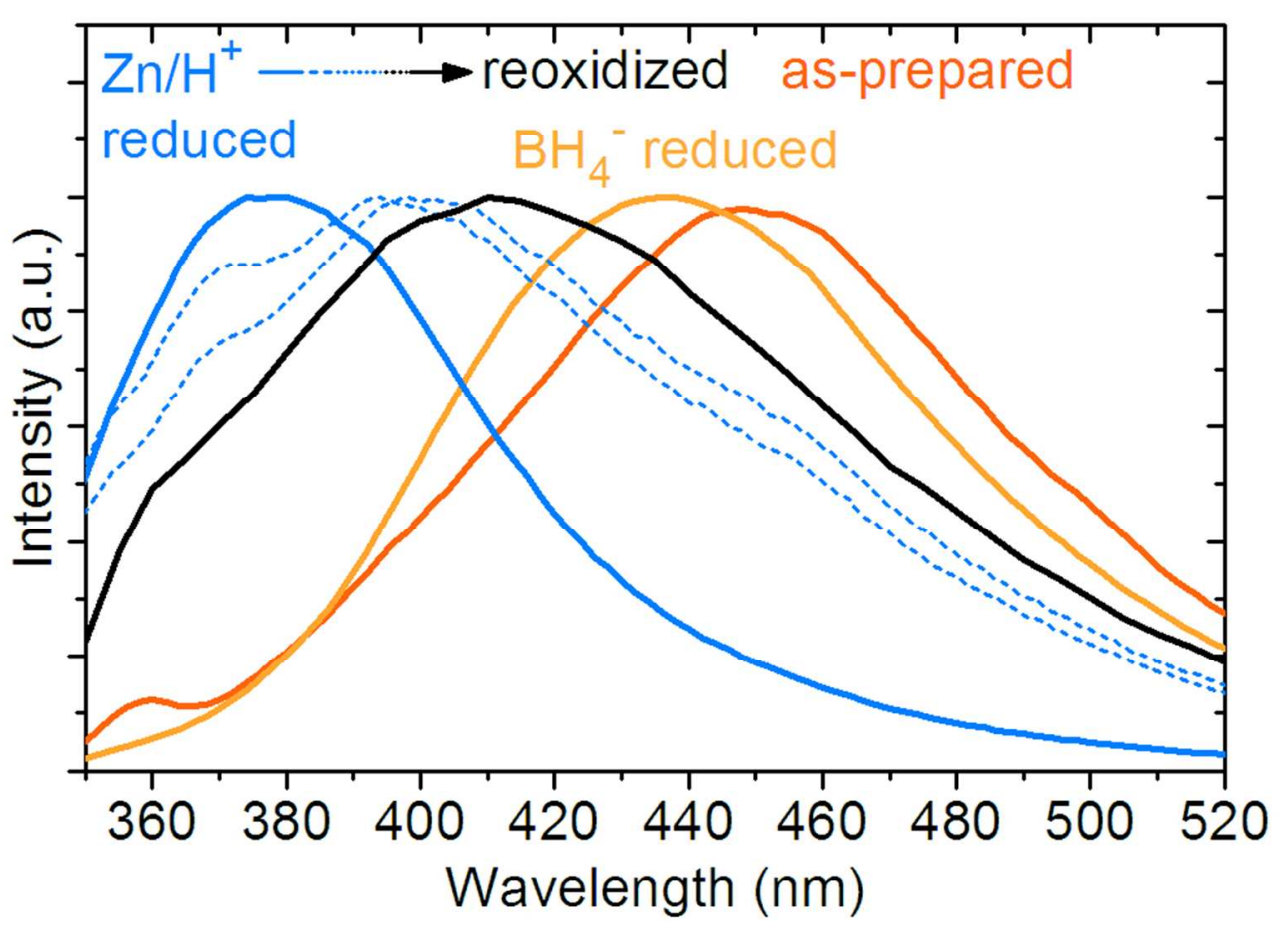

Figure 2.: Steady state PL spectra of different surface terminated SiC NCs. Excitation wavelength is $320 \mathrm{~nm}$. The emission maxima are at 455, 435, 380 and $410 \mathrm{~nm}$ for as-prepared, $\mathrm{BH} 4$ - reduced, $\mathrm{Zn} / \mathrm{H}+$ reduced and reoxidized samples, respectively. Blue dotted lines represent the emission upon oxidation of the $\mathrm{Zn} / \mathrm{H}+$ reduced sample as a function of illumination time from left to right. The final spectral shape is reached after 2-hour illumination (black line) using $320 \mathrm{~nm}$ excitation.

\section{$85 \times 59 \mathrm{~mm}(300 \times 300$ DPI $)$}


Figure 3. TRANES curves of the as-prepared, $\mathrm{BH} 4$ reduced and the reoxidized form $\mathrm{Zn} / \mathrm{H}+$ reduced samples. The $y$-axis is the fluorescence intensity in arbitrary unit. The zero levels vary from sample to sample. This way of representation was chosen for the sake of visibility. Notice that the plotted time intervals are $0.5 \mathrm{~ns}$ to $3.2 \mathrm{~ns}$ for the as-prepared and for the $\mathrm{BH} 4$ reduced samples, while the plotted period is $2.5 \mathrm{~ns}$ to $15 \mathrm{~ns}$ for the reoxidized from $\mathrm{Zn} / \mathrm{H}+$ reduced sample. In the case of the reoxidized sample the shorter wavelength and shorter lifetime emission center gives more intensive emission, thus TRANES yields the isoemissive point on a longer time scale. Figure $\mathrm{S} 6$ shows the DAS for the three samples.

$85 \times 119 \mathrm{~mm}(300 \times 300 \mathrm{DPI})$ 


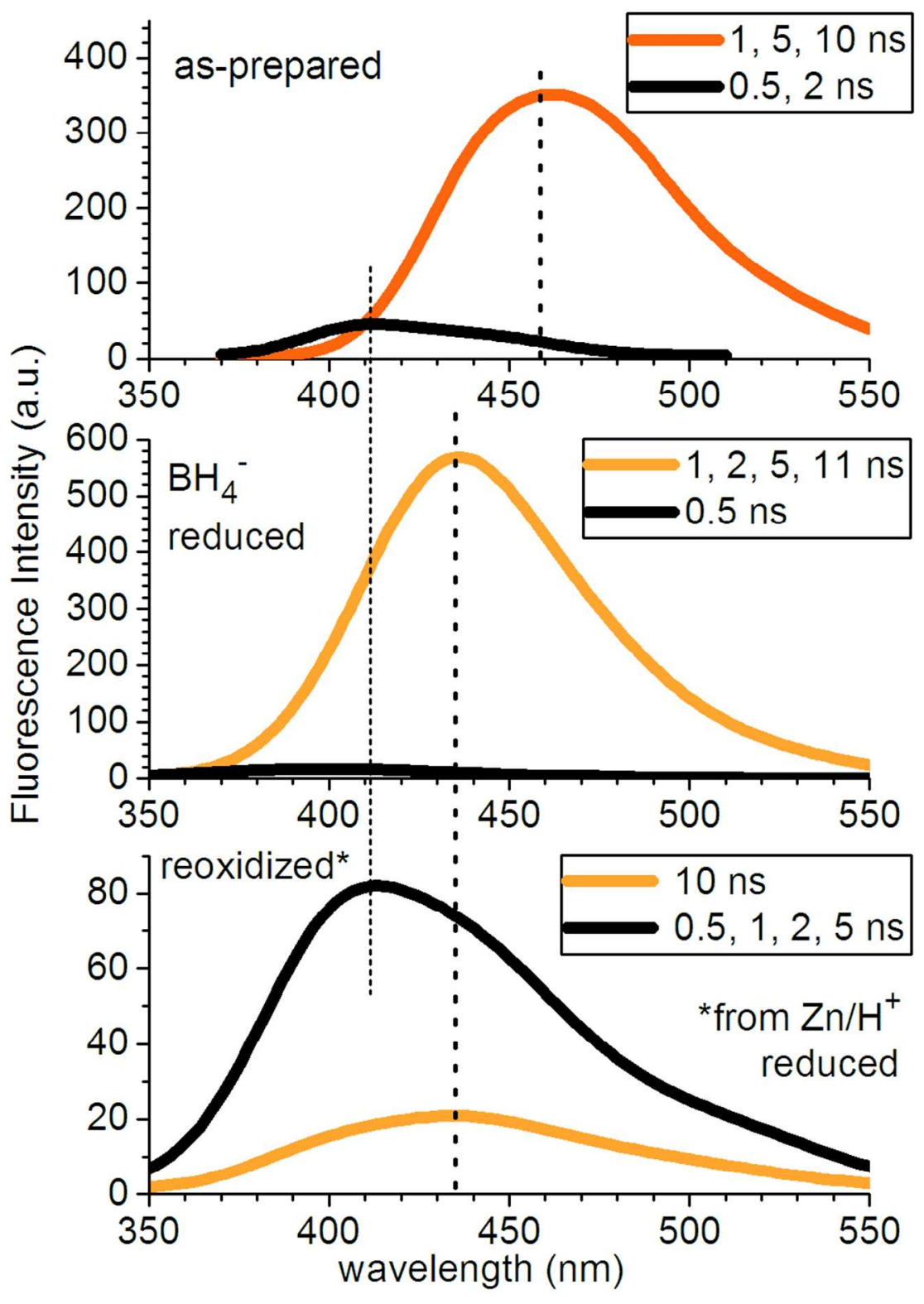

Figure 4.: Decay associated spectra (DAS) of as-prepared and reduced samples. Spectra having different lifetime components but the same maxima are weighted together for the sake of visibility. DAS confirms two emission centers in SiC NCs. The emission at $\sim 410 \mathrm{~nm}$ is due to the presence of fluorescent defects on the oxidized surface. $85 \times 119 \mathrm{~mm}(300 \times 300$ DPI) 


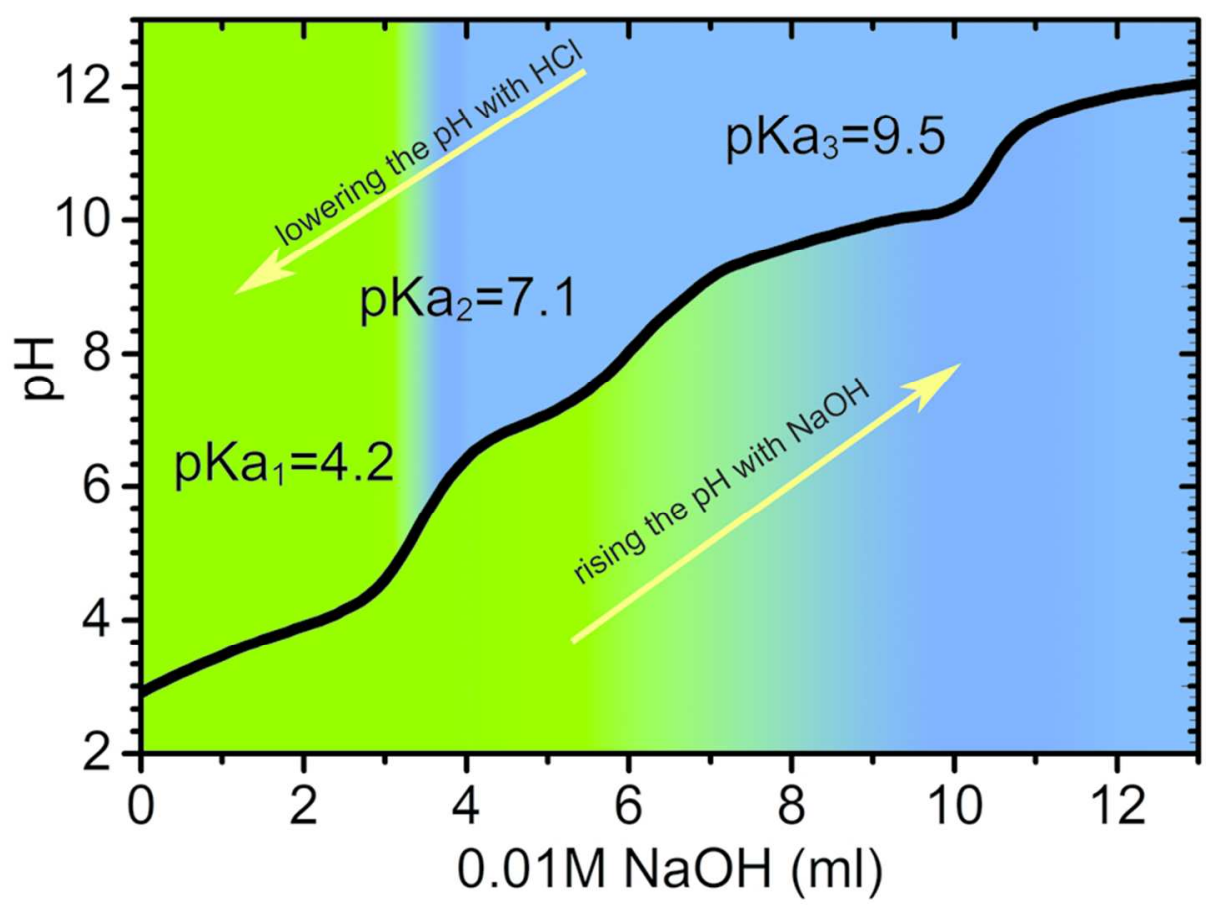

Figure 5.: Titration curve of SiC NCs in the $2-13 \mathrm{pH}$ range. Color changes below the titration curve represent the color changes of the solution when pH was changed from 2 to 13 . Color changes above the curve represent the color changes of the solution when $\mathrm{pH}$ was changed from 13 to 2 . $85 \times 60 \mathrm{~mm}(300 \times 300 \mathrm{DPI})$ 

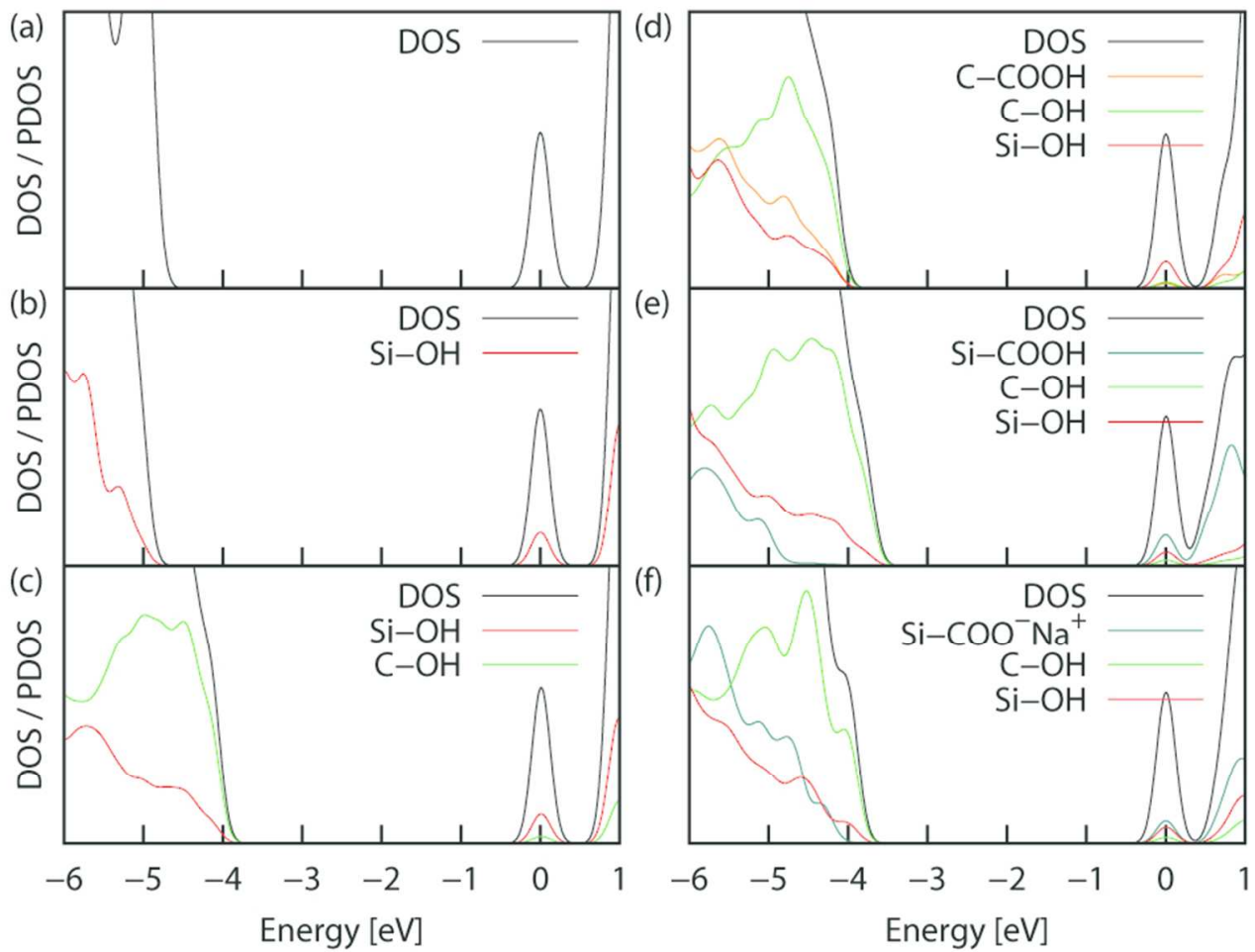

Figure 6.: Density of states (DOS) and projected density of states (PDOS) of our model SiC nanocrystal with different surface terminations: (a) hydrogenated surface, (b) " $\mathrm{Zn} / \mathrm{H}+$ " reduced and (c) BH4 re-duced. (d, e) DOS/PDOS of the model related to the "as prepared" sample, where the carboxyl groups bond to the C- and Si-terminated surface of the nanocrystals, respectively. (f) DOS/PDOS for the model of the sample with added $\mathrm{NaCl}$ where the carboxyl groups are dissociated. For the sake of easier com-parison, the DOS/PDOS was shifted along the energy axis until the peak associated with the lowest un-occupied molecular orbital is at $0 \mathrm{eV}$. The DOS and PDOS were calculated with the PBE0 exchange-correlation functional and double- $\zeta$ polarized basis set. $82 \times 67 \mathrm{~mm}(300 \times 300 \mathrm{DPI})$ 


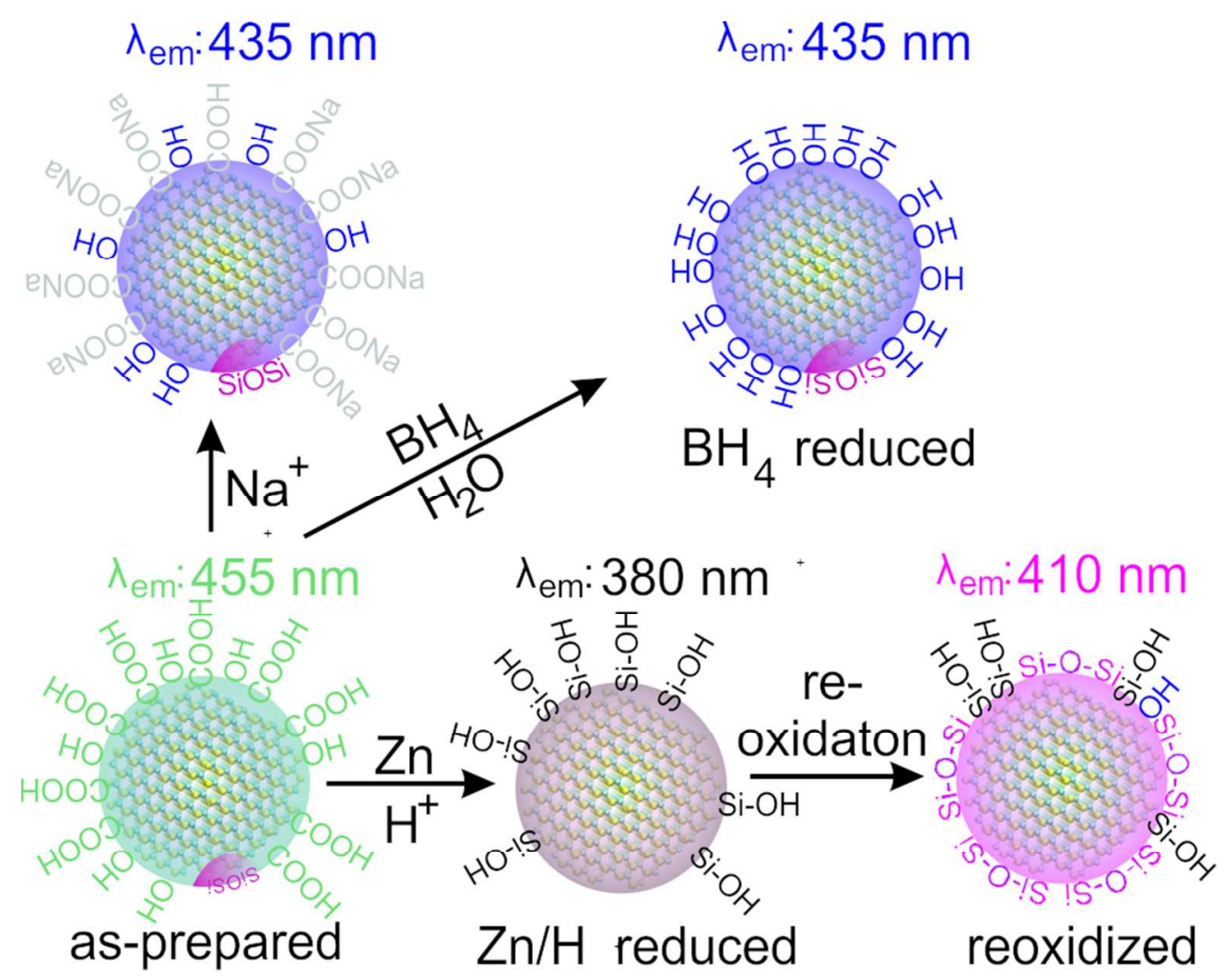

Scheme 1. Surface and environment dependent luminescence of SiC NCs $83 \times 66 \mathrm{~mm}(300 \times 300$ DPI $)$ 